\title{
Using forward calculations of the magnetic field perturbation due to a realistic vascular model to explore the BOLD effect
}

\author{
José P. Marques ${ }^{1,2 *}$ and R. W. Bowtell ${ }^{2}$ \\ ${ }^{1}$ Visual Neuroscience Laboratory, IBILI, University of Coimbra, Portugal \\ ${ }^{2}$ Sir Peter Mansfield Magnetic Resonance Centre, School of Physics and Astronomy, University of Nottingham, UK
}

Received 27 March 2007; Revised 6 August 2007; Accepted 18 September 2007

\begin{abstract}
This paper assesses the reliability of the infinite cylinder model used previously in the literature to simulate blood oxygenation level dependent (BOLD) signal changes. A three-dimensional finite element method was applied to a realistic model of the cortical vasculature, and the results compared with those generated from a simple model of the vasculature as a set of independent, randomly oriented, infinite cylinders. The realistic model is based on scanning electron microscopy measurements of the terminal vascular bed in the superficial cortex of the rat. Good agreement is found between the two models with regard to the extravascular $R_{2}{ }^{*}$ and $\mathrm{R}_{2}$ dependence on the cerebral blood volume and blood oxygenation fraction. Using the realistic model, it is also possible to gain further understanding of the relative importance of intravascular and extravascular BOLD contrast. A simple parameterisation of the dependence of the relaxation rates on relative cerebral blood volume and blood-tissue susceptibility difference was carried out, allowing discussion of the variation in the form of the haemodynamic response with field strength. Copyright (C) 2007 John Wiley \& Sons, Ltd.
\end{abstract}

KEYWORDS: 3D; three-dimensional; BOLD contrast; numerical simulations; BOLD parameterisation; realistic vasculature model

\section{INTRODUCTION}

Although functional magnetic resonance imaging (fMRI) based on blood oxygenation level dependent (BOLD) contrast (1) has become a widely used tool in neuroscience, the physiological changes that underpin the BOLD effect are still not completely understood. In particular, the relationship between the changes in blood oxygenation $(\Delta Y)$ and cerebral blood volume, occurring in active brain tissue, and the resulting variation in $T_{2}^{*}$ - or $T_{2}$-weighted images is not fully characterised. In probing this relationship, it has generally been assumed that the brain vasculature can be represented as an arrangement of randomly oriented, infinitely long cylinders of varying size (2-6). Here, we probe the effect of this assumption by using a three- dimensional (3D) finite element method to simulate BOLD signal changes produced by infinite

*Correspondence to: J. P. Marques, Laboratory of Functional and Metabolic Imaging, École Polytechnique Fédérale de Lausanne, Station 6, CH-1015 Lausanne, Switzerland.

E-mail: jose.marques@epfl.ch

Contract/grant sponsor: Fundacão para a Ciência e Tecnologia; contract/grant numbers: SFRH/BD/909/2000 and SFRH/BPD/20522/2004. Contract/grant sponsor: MRC; contract/grant number: G9900259.

Abbreviations used: BOLD, blood oxygenation level dependent; FFT, fast Fourier transform; FID, free induction decay; GE, gradient echo; ICM, infinite cylinder model; MION, monocrystalline iron oxide nanoparticle; PCV, packed cell volume; RVM, realistic vascular model; SE, spin echo. cylinders and a more realistic model of the human cortical vasculature. The latter is based on scanning electron microscopy measurements of the terminal vascular bed in the superficial cortex of the rat (7).

When simulating BOLD effects in fMRI, most researchers have centred their attention on the changes in blood oxygenation and volume in the brain vasculature when a region of the brain undergoes activation. Two approaches have generally been used:

- two-dimensional finite element numerical simulations of the magnetisation, considering an environment where the field inhomogeneity is generated by independent infinite cylinders, the internal magnetic susceptibility of which varies as the state changes between resting and activated $(3,5,8)$;

- analytical parameterisation of the problem, also assuming independent infinite cylinders, the BOLD response is calculated as a function of parameters such as blood volume, hematocrit (HCT), oxygen concentration, etc. $(4,6,9-11)$.

Parameter variations during activation can then be calculated (12) by trying to fit the simulated haemodynamic response function to a real BOLD response. Although the two approaches described above are methodologically very different, they employ a common assumption: the brain vasculature can be represented as an 
arrangement of independent, randomly oriented, infinite cylinders. Furthermore, intravascular and extravascular contributions to the contrast are largely discussed as independent mechanisms, and the literature for each of these mechanisms of contrast is vast, but not always well linked.

In this paper, the validity of the 'infinite cylinders' approach is assessed by evaluation of the results of 3D finite element simulations. Although an exact description of the vasculature for the whole human brain will never be available, the terminal vasculature bed in the superficial cortex of the rat, the morphology of which has been measured by scanning electron microscopy (7) (Fig. 1a), is a good starting point for addressing the validity of the infinite cylinder assumption. The aim is to find differences in signal behaviour predicted by

(i) the infinite cylinders model and (ii) a more realistic vascular distribution where, for example, the loss of coherence of extravascular and intravascular magnetisation results not only from the isotropic distribution of the angular orientations of the infinite cylinders, but also from diffusion in the field inhomogeneities generated by the ensemble of neighbouring vessels. The effect of water diffusion inside vessels, within which frequency shifts vary spatially, is also considered.

\section{METHODS}

The infinite cylinder model (ICM) and the realistic vascular model (RVM) were compared by simulating the evolution of magnetisation in the spatially varying magnetic fields generated by intravascular/extravascular susceptibility differences.

The ICM assumes that blood vessels are approximated by infinite cylinders, allowing a straightforward analytical solution of the problem, giving spatially varying frequency shifts, $\Delta \omega(\vec{r})$, for a single vessel exposed to a magnetic field, $B_{0}$ :

$$
\begin{aligned}
& \Delta \omega(\vec{r})=-\frac{1}{2} \gamma B_{0} \Delta \chi\left[\cos ^{2}(\theta)-\frac{1}{3}\right] \\
& \text { inside } \\
& \Delta \omega(\vec{r})=-\frac{1}{2} \gamma B_{0} \Delta \chi\left(\frac{R_{c y l i n d e r}}{r}\right)^{2} \sin ^{2}(\theta) \cos (2 \phi) \\
& \text { outside }
\end{aligned}
$$

Thus, the frequency shift due to such a vessel depends on the ratio of the cylinder radius, $R_{c y l i n d e r}$, to the radial distance from the centre of the cylinder, $r$, the intravascular/extravascular susceptibility difference, $\Delta \chi$, the angle between the vessel and the magnetic field, $\theta$, and the angle between the projection of the position vector, $\eta$, and magnetic field vector in the plane perpendicular to the vessel, $\Phi$. The susceptibility of blood plasma is similar to that of surrounding tissue, so that the dominant susceptibility difference is between red blood cells and plasma/tissue. The assumption made for the intravascular susceptibility is that, after time averaging, the blood has an equivalent susceptibility, given by $\Delta \chi=\mathrm{HCT}(1-Y) \chi_{d H b}$, where HCT is the hematocrit, $Y$ is the fractional oxygenation, and $\chi_{d H \mathrm{~b}}$ is the susceptibility of deoxyhaemoglobin [which has been reported to have a value of $0.18 \mathrm{ppm}$ in cgs units (13), which corresponds to $2.26 \mathrm{ppm}$ in SI units]. The ICM was generated by considering an ensemble of individual cubes, each containing one vessel. The angle, $\theta$, between
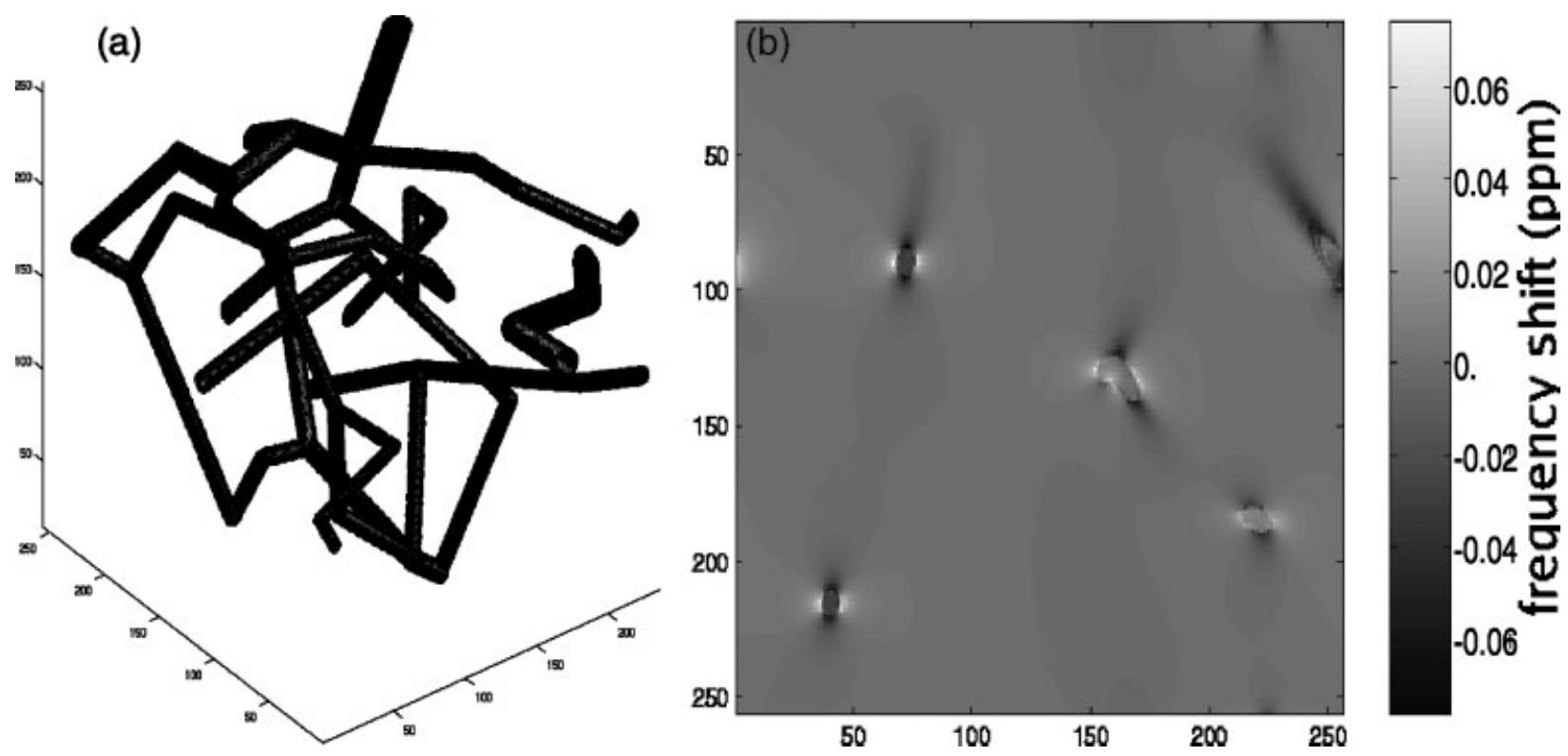

Figure 1. (a) Rat brain micro vasculature model obtained by scanning electron microscopy (7). The model shown here on a $256^{3}$ matrix spans a cube of the cortical bed with $150 \mu \mathrm{m}$ sides, incorporating 50 vessel segments that vary in diameter from 4 to $9 \mu \mathrm{m}$. (b) Magnetic field variation in ppm in a two-dimensional section calculated using the rat brain micro vasculature model for a blood-tissue susceptibility difference of $0.18 \mathrm{ppm}$. 
the vessel and the applied magnetic field varied across cubes, and ten different angles were considered. The signal evolution was simulated in each cube so as to form a free induction decay (FID). An average FID reflecting the signal from a voxel containing vessels with an isotropic distribution of orientation with respect to the field was formed by summing together the complex FIDs from the different cubes using a $\sin (\theta)$ weighting. Several length scales were also considered so that a wide range of vessel diameters was studied.

The RVM uses the distribution of magnetic susceptibility given by the rat brain microcirculation model (7) as a starting point. The model gives the coordinates of the different nodes of the vasculature and the radius of the cylinder connecting these nodes. This model spans a cube of the cortical bed with $150 \mu \mathrm{m}$ sides, incorporating 50 vessel segments with diameters that vary from 4 to $9 \mu \mathrm{m}$. The model was converted into a cubic $N \times N \times N$ matrix, $\operatorname{Subst}\left(r_{i, j, k}\right)$, with each element being labelled ' 1 ' or ' 0 ' depending on whether it corresponded to blood or grey matter (Fig. 1a). The frequency shift map was calculated (Fig. 1b) using a method described in the context of MRI $(14,15)$, in which the 3D fast Fourier transform (3DFFT) of the frequency offset is given by:

$$
\Delta \omega(\vec{k})=\frac{\gamma}{3}\left(\beta^{2}-1\right) \Delta \chi(\vec{k}) B_{0} \hat{z}
$$

where $\vec{k}$ is the coordinate position in $\mathrm{k}$-space, $\beta$ is the angle between the main magnetic field and $\vec{k}$, and $\Delta \chi(\vec{k})$ is the 3DFFT of the susceptibility distribution. Note that the result obtained with this method already contains the effect of the sphere of Lorentz, allowing an accurate calculation of intravascular frequency distributions for the RVM. Applying an inverse Fourier transform yields the frequency shift variation in real space. Several orientations of the model with respect to the static magnetic field and various length scales were again simulated.

Once the environment had been defined, the evolution of the magnetisation during a gradient echo (GE) or spin echo (SE) sequence was calculated by numerically applying the Bloch equations, taking into account the effects of relaxation, frequency shifts due to field inhomogeneities, and diffusion. At each time step, $\delta t$, the integrated effects (as opposed to the incremental effects, which yield a more stringent convergence criterion) of field inhomogeneity and relaxation on the transverse magnetisation, $M^{+}(\vec{r}, t)$, were evaluated using the Bloch equations:

$$
\begin{aligned}
& M^{+}(\vec{r}, t+\delta t) \\
& \quad=\left(M^{+}(\vec{r}, t) e^{i \gamma B(\vec{r}) \delta t}+\delta t D(\vec{r}) \nabla^{2} M^{+}(\vec{r}, t)\right) e^{-\delta t / T_{2}}
\end{aligned}
$$

where $D(\vec{r})$ is the diffusion coefficient, which is substance dependent.

The term reflecting the effect of diffusion has to be calculated at each time step, whereas $e^{i \gamma B(\vec{r}) \delta t}$ and $e^{-\delta t / T_{2}}$ may be calculated only once at the start of the simulation. The diffusion term was calculated using a finite difference solution in real space (16).

To make the problem tractable, the vessel walls were assumed to be impermeable. This assumption was implemented by imposing some restrictions on the simple finite difference solution. This involved simply inserting

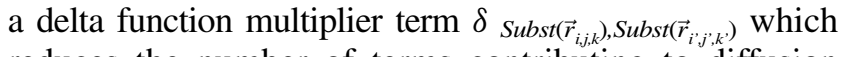
reduces the number of terms contributing to diffusion attenuation when the 'substance' of the neighbouring pixel is different. As substance changes only occur at blood/tissue boundaries, this modification makes the vessel walls effectively impermeable. Its implementation is described here for a one-dimensional case where diffusion is considered to only occur in the $x$ direction:

$$
\begin{aligned}
& \frac{M\left(\vec{r}_{i, j, k}, t+\delta t\right)-M\left(\vec{r}_{i, j, k}, t\right)}{\delta t} \\
& =\frac{D}{(\delta x)^{2}}\left[M\left(\vec{r}_{i-1, j, k}, t\right)\right. \\
& \left.\quad-M\left(\vec{r}_{i, j, k}, t\right)\right] \delta_{\text {Substr }\left(\vec{r}_{i, j, k}\right), \operatorname{Substr}\left(\vec{r}_{i-1, j, k}\right)} \\
& \quad+\frac{D}{(\delta x)^{2}}\left[-M\left(\vec{r}_{i, j, k}, t\right)\right. \\
& \left.\quad+M\left(\vec{r}_{i+1, j, k}, t\right)\right] \delta_{\text {Substr }\left(\vec{r}_{i, j, k}\right), \operatorname{Substr}\left(\vec{r}_{i+1, j, k}\right)}
\end{aligned}
$$

The maximum allowed time step, $\delta t$, is set by the largest value of the diffusion coefficient, $D_{\max }$, and the distance between neighbouring pixels $(\delta x, \delta y, \delta z)$, via $\delta \mathrm{t}<\left(\delta x^{2}+\delta y^{2}+\delta z^{2}\right) /\left(6 D_{\max }\right)$ or by the desired temporal resolution.

Calculations were carried out using two different regimes of blood/tissue susceptibility difference $(\Delta \chi)$, corresponding to the typical values occurring in the absence or presence of exogenous contrast agents. $\Delta \chi$ values in SI units of $0.45 \mathrm{ppm}$ (oxygenation fraction, $Y=0.5$ ), $0.36 \mathrm{ppm}$ (resting state, $Y=0.6$ ), $0.27 \mathrm{ppm}$ (intermediate state, $Y=0.7$ ) and $0.18 \mathrm{ppm}$ (activated state, $Y=0.8$ ) were used to simulate BOLD susceptibility changes in the absence of a contrast agent (these values were calculated using the relationship $\Delta \chi=\mathrm{HCT}$ $(1-Y) \chi_{d H b} ; \chi_{d H \mathrm{~b}}=2.26 \mathrm{ppm}$ in SI units and HCT $=$ $0.4)$. HCT for the case of exogenous contrast agents, such as monocrystalline iron oxide nanoparticle (MION) or gadolinium diethylenetriamine penta-acetic acid (DTPA), susceptibility differences of 1.41, 2.82, 4.23 and $5.64 \mathrm{ppm}$ (corresponding to MION concentrations in the blood of 1 , 2, 3 and $4 \mathrm{mM}$ ) were considered. These values are based on susceptometry studies using MRI described by Weisskoff and Kiihne (13).

Blood volume fractions of $1.29 \%, 2.56 \%, 3.76 \%$ and $4.90 \%$ were considered in the simulations. The value set by the form of the RVM was $1.29 \%$ and all the other values were obtained by multiplying all the vessel radii given in the original model by $\sqrt{ } 2, \sqrt{ } 3$ and $\sqrt{ } 4$. This 
meant that the volume changes considered here correspond to vessel dilation rather than recruitment (i.e. there is an associated change in vessel diameter). A similar procedure was used for the ICM.

Diffusion coefficients of $D_{B}=1.45 \times 10^{-9} \mathrm{~m}^{2} \mathrm{~s}^{-1}$ inside and $D_{G M}=0.7 \times 10^{-9} \mathrm{~m}^{2} \mathrm{~s}^{-1}$ outside vessels were assumed. For the SE simulation, the echo time was set to the $T_{2}$ of grey matter [the values used for relaxation times were $T_{2 G M}=80 \mathrm{~ms}$ and $T_{2 B}=100 \mathrm{~ms}$, which are similar to values at $3 \mathrm{~T}$ described in the literature $(17,18)]$. Several length scales were considered by simply rescaling the distance between neighbouring volume elements in the RVM or ICM matrix, so that a wide range of vessel diameters from capillaries $(4 \mu \mathrm{m})$ to large venules $(300 \mu \mathrm{m})$ could be studied.

Two different matrix sizes, $32^{3}$ and $64^{3}$, were used in representing the ICM in order to test whether reduced spatial resolution would be an issue when simulating the $\mathrm{RVM}$, as a $128^{3}$ matrix was the largest that could be simulated in an acceptable time $(30 \mathrm{~h})$. Simulations of the ICM in a $32^{3}$ matrix were also performed using field maps calculated via the FFT-based approach (14) rather than the analytical method, to test whether the FFT method introduced any intrinsic differences. No significant differences in the simulated ICM signal behaviour were observed when reducing the matrix size or calculating the field shift with the FFT method rather than analytically, leading us to believe that any differences in the results between ICM and RVM models are not due to the methodology used, but to the model under study.

The simulations spanned a range of intravascular/ extravascular susceptibility differences, blood volume and vessel sizes, potentially allowing the overall behaviour of the relaxation processes to be parameterised. Such parameterisation is valuable, as it allows evaluation of BOLD and contrast agent effects over a wide range of field strengths.

\section{Extravascular contrast}

Intravascular and extravascular compartments were studied separately. Extravascular signal in GE experiments was characterised by two different models (19).

(i) Considering that the extravascular distribution of field values $B_{e x t}$ can be represented by a Cauchy distribution

$p\left(B_{\text {ext }}\right)=\frac{1}{\sqrt{\pi}} \frac{\sigma_{B}}{\sigma_{B}^{2}+B_{\text {ext }}^{2}}$

where $\sigma_{\mathrm{B}}$ characterises the width of the distribution of field shifts, $p\left(B_{\text {ext }}\right)$. The signal decay in the presence of such a field distribution will be given by $G(t) \propto \exp \left(-t \gamma \sigma_{B}\right)$, leading to a relaxation rate $R_{2}^{*}=\gamma \sigma_{\mathrm{B}}$ in the static regime where diffusion has no effect. (ii) Considering a Gaussian field distribution

$$
p\left(B_{\text {ext }}\right)=\frac{1}{\sqrt{2 \pi \sigma_{B}^{2}}} e^{-B_{\text {ext }}^{2} /\left(2 \sigma_{B}^{2}\right)}
$$

for which it can be shown that the signal decay is characterised by (19),

$$
G(t)=e^{\left[-\left\langle\Delta \omega^{2}\right\rangle \tau_{c}^{2}\left(e^{-\frac{t}{\tau_{c}}}-1+\frac{t}{\tau_{c}}\right)\right]}
$$

where $\left\langle\Delta \omega^{2}\right\rangle=\gamma^{2} \sigma_{B}^{2}$ is the mean square frequency deviation of the Gaussian field distribution, and $\tau_{\mathrm{c}}$ is the corresponding correlation time for field fluctuations produced by diffusion in spatially varying fields. When $\tau_{c}$ is very large (no diffusion approximation), the signal decay naturally becomes Gaussian.

Fitting of simulated GE data to these two different models was accomplished by applying least squares minimisation to the signal decay. In the case of the Gaussian distribution, characterised by eqn (5), simulated data spanning times from $t=0$ to $t=T_{2 G M}$ were considered, but, in the case of the Cauchy distribution (i), fitting was only applied to data spanning times from $t=20 \mathrm{~ms}$ to $t=T_{2 G M}$ so as to avoid the initial nonexponential decay (20). Other models are available in the literature such as those described in refs $(4,21,22)$, which could equally well have been used at this stage. One of the most extensively studied models is that of Yablonskiy and Haacke (4). This was not used in this study because of the additional complexity of explicitly separating the quadratic and linear exponential decays (20).

In simulating SE signals, time constraints meant that it was only possible to consider the application of a single $180^{\circ} \mathrm{RF}$ pulse. It therefore had to be assumed that the SE was described by an exponential decay with a time constant $R_{2}$ calculated using only two data points at times $t=0$ and $t=T_{2 G M}$.

The dependence of the parameters $\left(\left\langle\Delta \omega^{2}\right\rangle, \tau_{c}, R_{2}\right.$ and $R_{2}^{*}$ ) on the total blood volume fraction, $V$, and the magnetic susceptibility, $\chi$, for different length scales was calculated by fitting the parameters to an expression of the form:

$$
\alpha V^{\beta} \chi^{\gamma}
$$

Such an expression for characterising relaxivity was initially proposed by Fisel et al. (23). It is worth noting that the power of $\chi$ also effectively characterises the dependence of relaxivity on the $B_{0}$ field, as all the expressions could be rewritten as $\alpha V^{\beta} \chi^{\gamma}\left(\frac{B_{0}}{3}\right)^{\gamma}$. The values reported hereafter for $V$ and $\chi$ are always in percentage of volume (\%) and parts per million (ppm) respectively.

In the case of exponential signal decay (i), it is possible to consider the total effect of a distribution of vessel diameters as being simply the sum of the relaxation rate contributions due to vessels of different sizes $(6,21)$, which we assume can be written as $R_{2 \text { comb }}=$ $R_{2 G M}+\sum_{i} \alpha_{i} V^{\beta_{i}} \chi^{\gamma_{i}}$. The validity of this approach is 
based on the observation that the number of vessels, $N(d)$, with a specific diameter, $d$, in the brain scales as $1 / d^{3}(24)$, while the blood volume corresponding to the RVM scales with $d^{3}$. This means that the volume fraction is independent of $d$, so that the value of $V$ is the same for contributions to the overall relaxation rate from vessels of different sizes. In this approach, the effect of capillaries is considered to act as a background effect on the relaxation rate around venules, while these act as background for relaxation around larger veins. Here, we combined the relaxation rate contributions from RVM with three different average vessel sizes (7, 21 and $63 \mu \mathrm{m})$. Consequently, at the smallest length scales, $S$ (corresponding to vessel diameters ranging from $5 \mu \mathrm{m}$ to $12 \mu \mathrm{m}$ in the RVM), the decay of the signal from grey matter is described by $e^{-t R_{2, S}}$. At an average length scale, $M$ (corresponding to vessel diameters ranging from $15 \mu \mathrm{m}$ to $36 \mu \mathrm{m})$, the transverse magnetisation of grey matter, which is affected by field inhomogeneity caused by these vessels, is already decaying at $e^{-t R_{2, S}}$, and therefore the final relaxation of such grey matter will be described by $e^{-t R_{2, M}} e^{-t R_{2, S}}$. Finally for the larger vessels, with diameters ranging from $45 \mu \mathrm{m}$ to $108 \mu \mathrm{m}$, using the same reasoning, the grey matter decay inside the voxel that contains all length scales will be given by, $e^{-t R_{2, L}}$ $e^{-t R_{2, M}} e^{-t R_{2, S}}$. Therefore, the signal decay can be rewritten as, $e^{-t\left(R_{2, L}+R_{2, M}+R_{2, S}\right)}$. In combining the relaxation rates, we assumed that the blood volume fraction due to each model takes the same value, $V$, so that the final combined model has a total blood volume fraction of $3 \mathrm{~V}$. A similar approach was adopted for the ICM, but in this case three discrete vessel sizes were considered.

\section{Intravascular contrast}

There are various contributions to the intravascular signal decay, including: (a) phase variation due to the distribution of cylinder orientations; (b) dephasing resulting from field inhomogeneities generated by neighbouring vessels; (c) contribution due to blood flow and diffusion inside connected vessels that have different orientations, which is not calculable with the simple ICM.

(a) The intravascular FID from a large number of isotropically oriented infinite cylinders containing a substance with susceptibility, $\chi$, is the integral over the entire solid angle of the individual FIDs of the substance inside each cylinder, $e^{i \Delta \omega(\vec{r}) t}$, where $\Delta \omega(\vec{r})$ is given by eqn (1). The integral can be written as,

$$
I(t)=\frac{S_{0}}{2} \int_{0}^{\pi} \sin \theta e^{i k\left(3 \cos ^{2} \theta-1\right) t} d \theta
$$

where $k={ }_{3}^{2} \pi \quad \gamma B_{0} \chi$ (4). This expression can be simplified by using the relationship, $\left(3 \cos ^{2} \theta-1\right)=3 / 2 \cos ^{2} \theta+3 / 2, \quad$ and then expanding the exponential of the integrand using $\mathrm{e}^{i x \cos \theta}=J_{0}(x)+\sum_{n=1}^{\infty} 2 i^{n} J n(x) \cos n \theta$, leading to a decay of the form:

$$
I(t)=S_{0} e^{i 3 k t / 2}\left(J_{0}(3 k t / 2)-2 \sum_{n=1}^{\infty} i^{n} \frac{1}{4 n^{2}-1} J_{n}(3 k t / 2)\right)
$$

(b) The decay resulting from dephasing due to fields generated by neighbouring vessels can be analysed using the histogram of field values in the intravascular compartment.

(c) The third term can be assessed by considering the effect of diffusion in the gradients present inside the vessels as an exponential perturbation to eqn (8).

Here, the effect of erythrocytes and surrounding plasma, which is dominant over the mechanism listed above, is not considered. This is due to the lack of spatial resolution to include erythrocytes and to the fact that such contrast can be studied with greater accuracy in vitro (18).

\section{RESULTS}

\section{Extravascular contrast}

To help in understanding the extravascular relaxation processes, it is useful first to visualise the frequency shift distributions produced using RVM and ICM. Figure 2a,b shows the field histograms for various values of partial blood volume using the two different models. The field distributions shown in Fig. 2a,b are very similar in form and range of values, indicating that the ICM is generally a good model for characterising the extravascular field distribution. The best fit to the RVM data was achieved using a product of a Gaussian and a Cauchy distribution. The low frequencies are best characterised by the Cauchy distribution, whereas the Gaussian distribution characterises the high frequencies. It is interesting to note that the width parameter of the Gaussian distribution remains constant as the blood volume fraction changes, whereas the width of the Cauchy distribution increases linearly with volume fraction. This is foreseeable, as the Gaussian distribution characterises the highest frequency variations, and the maximum frequency shift generated by a cylinder is independent of its radius. As would be expected, it was found that both widths increased linearly with $\chi$. The shape of the histogram of extravascular frequency distribution will have a direct relationship to the form of the extravascular FID. In the absence of diffusion, this relationship is the Fourier transform. The use of the product of a Gaussian and a Cauchy distribution to characterise the frequency distribution is purely 

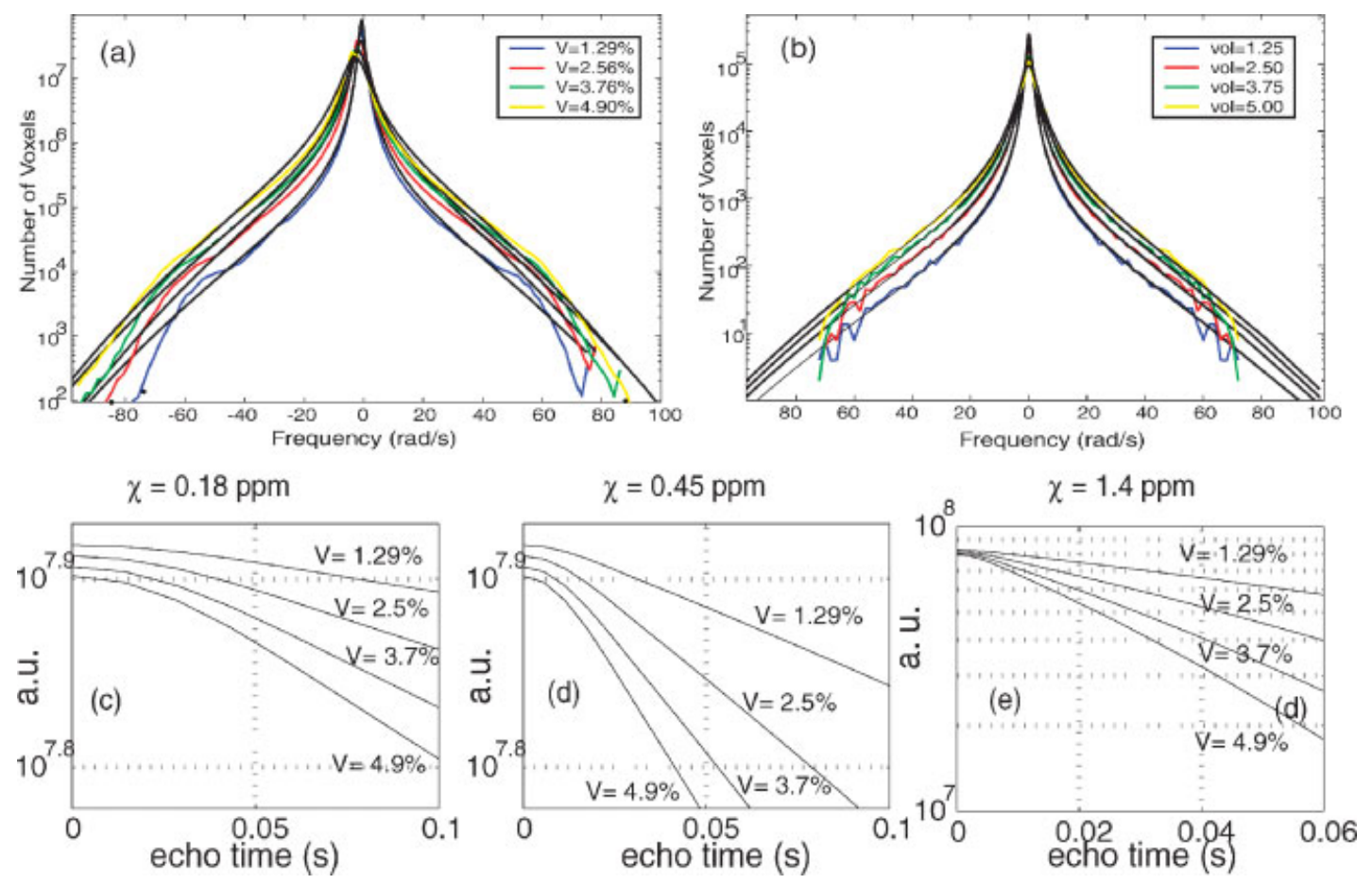

Figure 2. Distributions of the frequency shift in grey matter for (a) RVM and (b) ICM considering a susceptibility difference of $0.18 \mathrm{ppm}$ between intravascular and extravascular regions for partial volumes of $1.29 \%$ (blue), $2.56 \%$ (red), $3.76 \%$ (green) and $4.90 \%$ (yellow) at a $B_{0}$ field of 3 T. The histograms were best fitted to a product of a Cauchy distribution and a Gaussian distribution (black lines). Simulated extravascular signal FID for the RVM at different blood volumes simulated at a $B_{0}$ field of $3 \mathrm{~T}$ shown in a logarithmic plot. Susceptibility differences between vessels and grey matter of (c) 0.18 , (d) 0.45 and (e) 1.4 were considered.

phenomenological and leads to an FID formed from the convolution of a Gaussian exponential decay. FIDs generated by Fourier transformation of the field distributions are shown in Fig. 2c-e for various volume fractions and susceptibility differences at $3 \mathrm{~T}$. It can be seen that the curves have different forms in the different regimens: (c) for low intravascular/extravascular susceptibility differences as found in the activated brain, the decay is non-exponential at short echo times; (d) but such non-exponential behaviour does not occur at high concentrations of deoxyhaemoglobin for echo times that would typically be used in functional MRI [these observations are in agreement with ref. (4)]. For low and high concentrations of paramagnetic contrast agents, such as MION (e), the data are well characterised by a single exponential decay time constant.

Figure 3 shows the dependence of the relaxation rate changes $\left(\Delta R_{2}\right.$ and $\left.\Delta R_{2}{ }^{*}\right)$ due to BOLD effects in SE and GE data as a function of the average vessel diameter calculated over all the vessels present in the RVM. It can be seen that, although the values for the two different models are not coincident, the dependence on vessel diameter is very similar. This dependence means that in SE BOLD experiments, the capillaries and small venules are the dominant source of contrast, whereas the main contribution to contrast in GE experiments comes from venules and veins, as has previously been reported $(3,5)$.
Although the relaxation rate changes are smaller in the ICM than in the RVM, part of the discrepancy shown in Fig. 3 results from the inability to perfectly match the partial volume of the ICM to that of the RVM because of the limited spatial resolution in the $64 \times 64 \times 64$ matrix used to simulate the ICM.

Figure 4 shows the parameters that best fit the $R_{2}{ }^{*}$ and $R_{2}$ values of the extravascular signal as a function of the vessel diameter (in the case of the RVM, the average diameter present in the model is used); it is
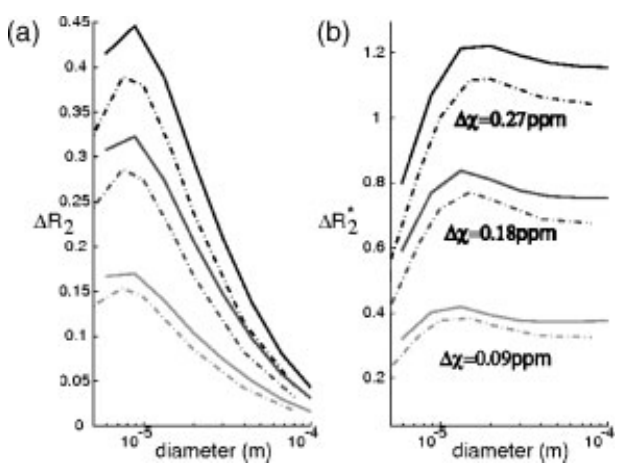

Figure 3. Variation (a) $\Delta R_{2}$ and (b) $\Delta R_{2}{ }^{*}$ for partial volumes of $1.25 \%$ and $1.29 \%$ (for the ICM and the RVM respectively) due to a change in blood susceptibility, $\Delta \chi$, as a function of the vessel diameter. Solid lines show the values obtained using the RVM, and the dashed lines represent values obtained using the ICM. 

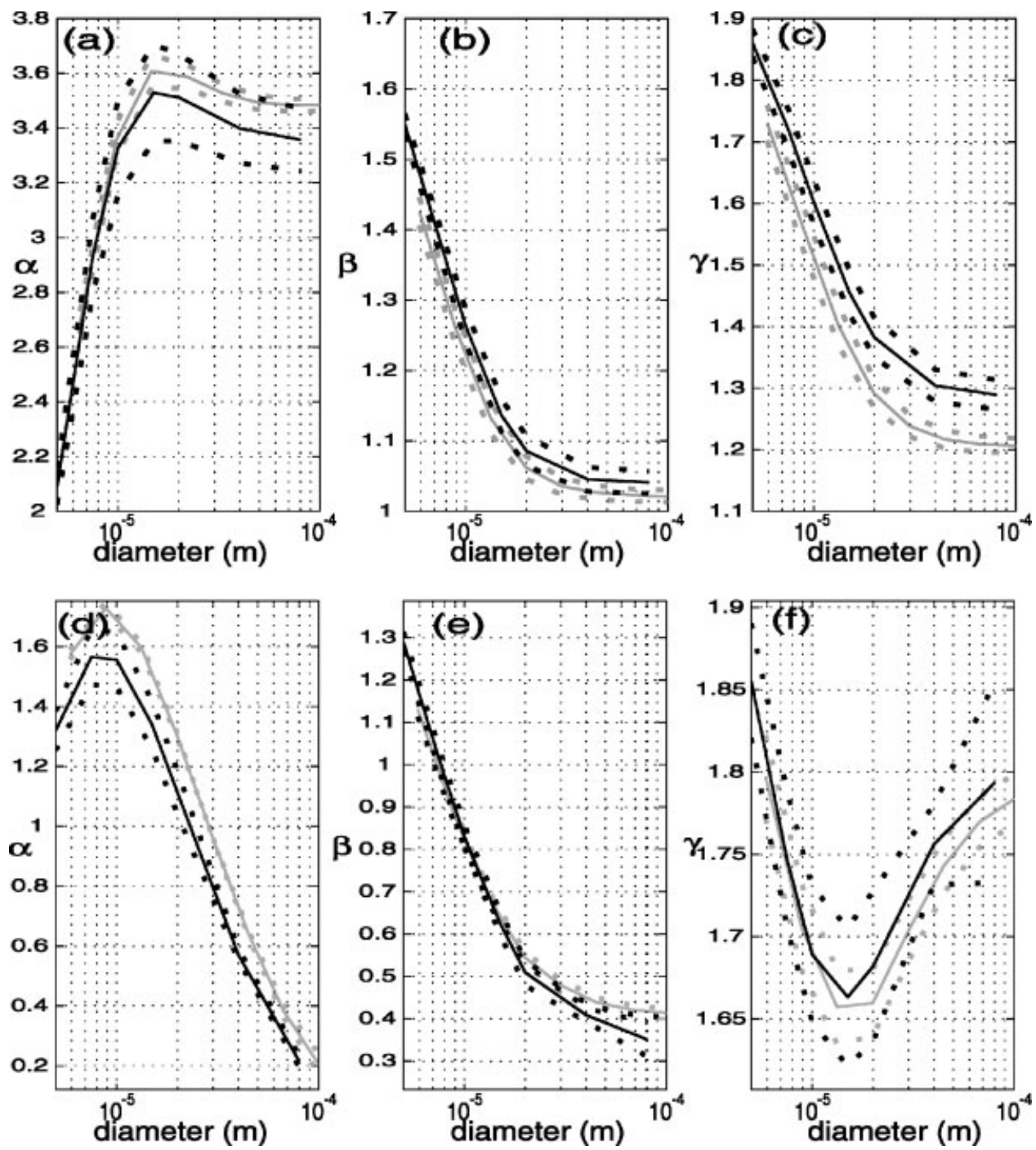

Figure 4. Variation in the parameters $(a, d) \alpha,(b, e) \beta$ and $(c, f) \gamma$ (continuous lines) with the average vessel diameter obtained for the ICM (black) and the RVM (grey). In each case $R_{2}{ }^{*}$ $(\mathrm{a}, \mathrm{b}, \mathrm{c})$ and $R_{2}(\mathrm{~d}, \mathrm{e}, \mathrm{f})$ values obtained for varying values of $V$ and $\chi$ were fitted to the equation $R_{2}^{*}=R_{2, G M}+\alpha V^{\beta} \chi^{\gamma}$ and $R_{2}=R_{2, G M}+\alpha V^{\beta} \chi^{\gamma}$ respectively. Dotted lines indicate standard deviations.

possible to observe the similarity of the dependence of the parameters obtained for the ICM and RVM. The parameter that most significantly differs between the two models, for both GE and SE data, is the proportionality constant $\alpha$, which is $\sim 10 \%$ higher for the RVM.

Vessels with a large range of diameters will generally be present in any voxel. Considering vessel sizes, $d$, ranging from $4 \mu \mathrm{m}$ to $110 \mu \mathrm{m}$ where all the vessels have a number density $\propto 1 / d^{3}(24)$, the combined dependence on the percentage blood volume fraction and blood susceptibility for the RVM was found to be:

$$
\begin{aligned}
& R_{2 R V M}=R_{2 G M}+1.23( \pm 0.04) V^{0.87( \pm 0.01)} \Delta \chi^{1.74( \pm 0.02)} \\
& R_{2 R V M}^{*}=R_{2 G M}+2.73( \pm 0.07) V^{1.13( \pm 0.01)} \Delta \chi^{1.29( \pm 0.01)}
\end{aligned}
$$

Copyright (C) 2007 John Wiley \& Sons, Ltd. whereas for the ICM, the aggregate effect was found to be:

$$
\begin{aligned}
& R_{2 I C M}=R_{2 G M}+0.88( \pm 0.04) V^{0.98( \pm 0.02)} \Delta \chi^{1.79( \pm 0.02)} \\
& R_{2 I C M}^{*}=R_{2 G M}+2.49( \pm 0.09) V^{1.15( \pm 0.01)} \Delta \chi^{1.38( \pm 0.02)}
\end{aligned}
$$

Figure 5 shows the parameterisaton of $\tau_{\mathrm{c}}$ and $\left\langle\Delta \omega^{2}\right\rangle$ obtained using eqn (5). Although the fits are more accurate even considering that the degrees of freedom are reduced because of the use of one extra fitting parameter, it should be noted that, in Fig. 5, the proportionality constant, $\alpha$ [see eqn (6)], for $\tau_{\mathrm{c}}$ becomes smaller as the length scale (diameter of vessels) increases, when it would be expected to increase. As a consequence, these parameters may be used to predict the signal decay at different field strengths, length scales, susceptibility 

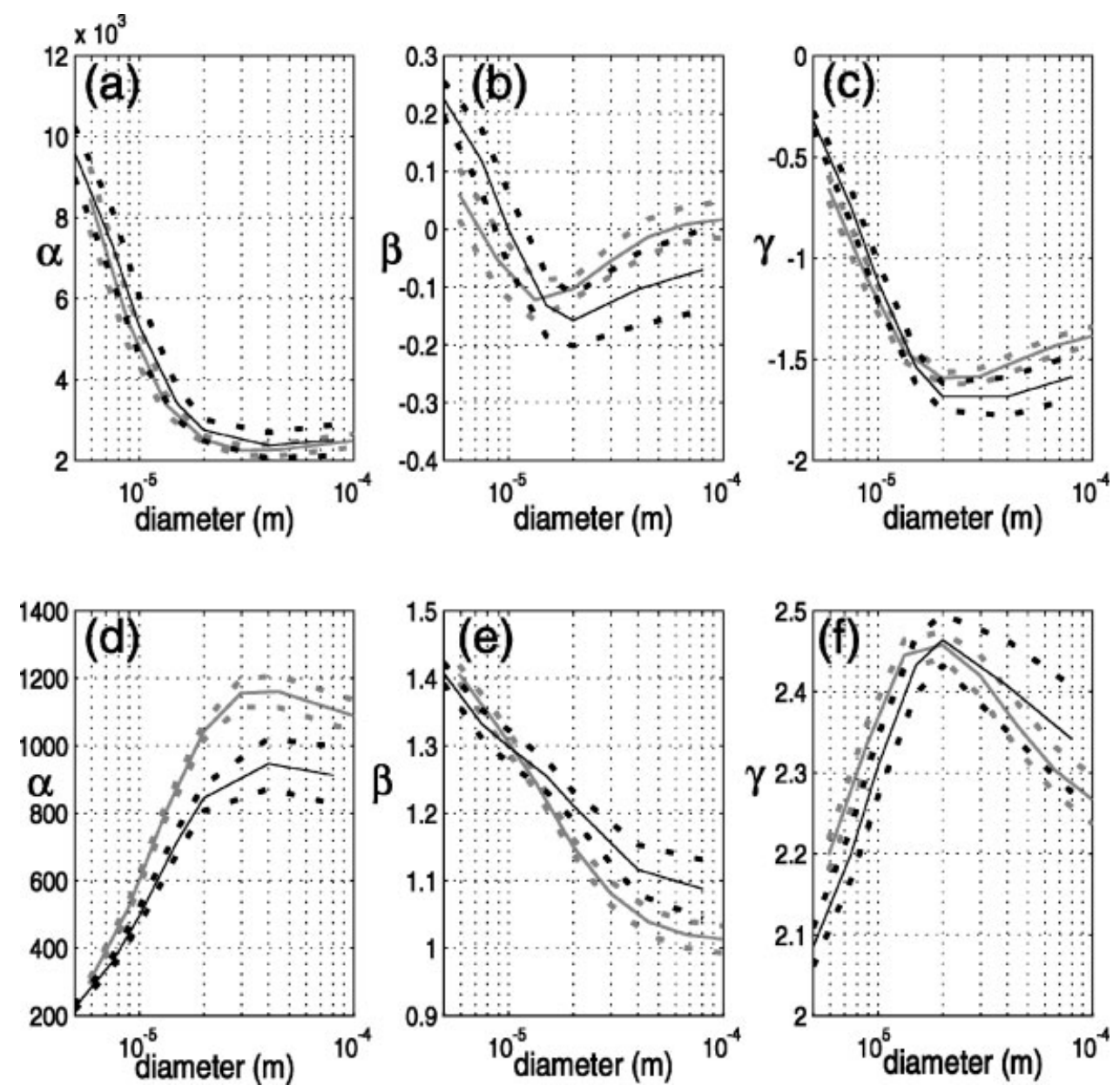

Figure 5. Variation in the parameters $(a, d) \alpha,(b, e) \beta$ and $(c, f) \gamma$ (continuous lines) with the average vessel diameter obtained for the ICM (black) and the RVM (grey) that characterise $\tau_{c}$ and $\left\langle\Delta \omega^{2}\right\rangle$. In each case, $\tau_{c}$ and $\Delta \omega^{2}$ values obtained for varying values of $V$ and $\chi$ were fitted to the function $\alpha V^{\beta} \chi^{\gamma}$. Dotted lines indicate standard deviations.

differences, and partial volumes, but it may be misleading to give them too much physical relevance. Nevertheless, it is tempting to remark that the dependence of the parameter $\left\langle\Delta \omega^{2}\right\rangle$ on the partial volume (approximately linear) and susceptibility (quadratic dependence) could be predicted from analysing the parameters characterising the fits to the distributions of Fig. 2a,b, as could the insensitivity of $\tau_{\mathrm{c}}$ to variations in the volume fraction and its dependence on the susceptibility. The negative power means that $\tau_{\mathrm{c}}$ decreases with the increasing $\chi$.

Figure 6 shows the variation with vessel size of the parameters characterising the relaxation rate, $R_{2}{ }^{*}$, of extravascular signal for values of susceptibility difference relevant to intravascular contrast agents. The exponential fitting used to identify $R_{2}{ }^{*}$ was carried out in the early portion of the signal variation with echo time that corresponds to the mono-exponential decay shown in Fig. 2e. The parameter values are significantly different from those found for typical deoxyhaemoglobin susceptibility differences at $3 \mathrm{~T}$ (top row of Fig. 4). In particular,
$R_{2}{ }^{*}$ is found to vary linearly with both the partial volume and susceptibility difference. The different dependences are partially related to the more adequate fitting of the exponential decay for higher susceptibility differences, as the initial non-exponential decay is absent.

Figure $7 \mathrm{a}$ shows the effect that the change in the susceptibility and blood volume fraction on activation has on extravascular $\Delta R_{2}{ }^{*}$ for the RVM (grey contour lines for $3 \mathrm{~T}$ and black contour lines for $9 \mathrm{~T}$ ). The haemodynamic response can be represented as a path on this surface (black line). Negative values of $\Delta R_{2}{ }^{*}$ cause a positive BOLD response, and a negative BOLD response is caused by a positive change in relaxivity. The generation of a negative BOLD response using the model $R_{2}{ }^{*}=\alpha V^{\beta} \chi^{\gamma}$ implies that changes in volume, $\Delta V$, and susceptibility, $\Delta \chi$, obey the inequality, $\beta \Delta V / V+\gamma \Delta \chi / \chi$. Such a negative BOLD response, generally occurring as an initial dip, has mainly been reported at high fields (25) and is elusive, but not undetectable at lower fields (26). The initial dip is expected to be mainly localised in the 

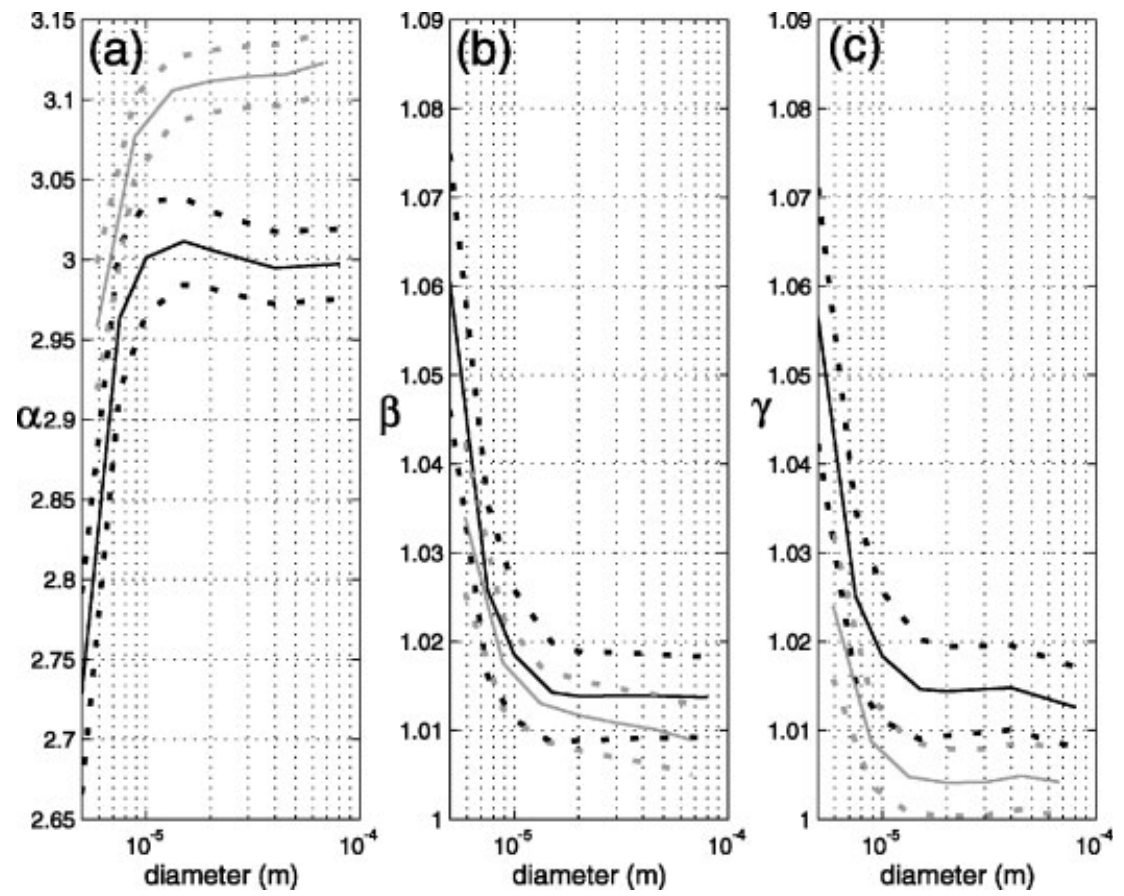

Figure 6. Variation in the parameters (a) $\alpha$, (b) $\beta$ and (c) $\gamma$ (continuous lines) with the average vessel diameter obtained for the ICM (black) and the RVM (grey). In each case $R_{2}{ }^{*}$ values obtained for various values of $V$ and $\chi$ (in a range corresponding to exogenous contrast agents) were fitted to the equation $R_{2}{ }^{*}=R_{2, G M}+\alpha V^{\beta} \chi^{\gamma}$. Dotted lines indicate standard deviations.

capillaries. At $3 \mathrm{~T}$, the condition for negative BOLD response to occur is $1.4 \frac{\Delta V}{V}+1.7 \frac{\Delta \chi}{\chi}>0$, whereas, at the higher fields of $9 \mathrm{~T}$, the negative BOLD condition is $1.03 \frac{\Delta V}{V}+1.02 \frac{\Delta x}{\chi}>0$ (Figs. 4 and 6). The condition for the initial dip to occur with an increase in blood volume fraction is less restrictive at high field $\Delta \chi>-1.01 \frac{\chi}{V} \Delta V$, than it is at low fields, $\Delta \chi>-0.82 \frac{\chi}{V} \Delta V$.
A possible haemodynamic response curve is shown in Fig. 7. This curve was simulated using the balloon model as described by Buxton et al. (27). The parameters used in equations $10-14$ of that paper were: $\delta_{f}=2 \mathrm{~s}, \delta_{m}=6 \mathrm{~s}$, $\tau_{h}=1.3 \mathrm{~s}, \tau_{M T T}=4 \mathrm{~s}, \tau^{-}=20 \mathrm{~s}, \tau^{+}=10 \mathrm{~s}, f_{1}=m=1.5$.

Figure $7 \mathrm{~b}$ shows the variation in the BOLD signal change with time for the two different regimes, making it (a)

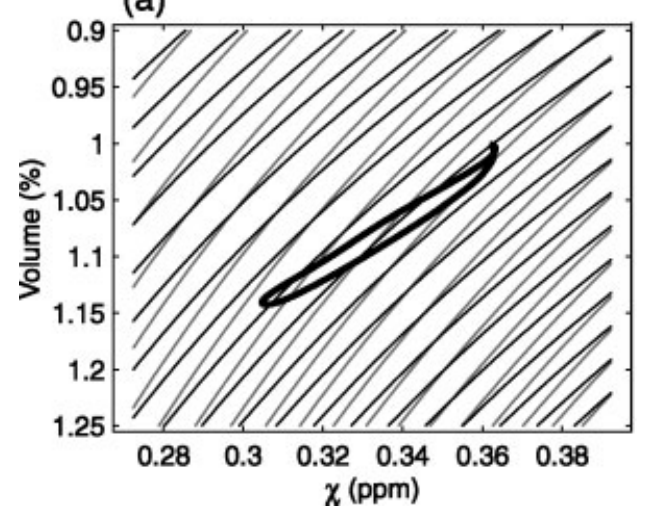

(b)

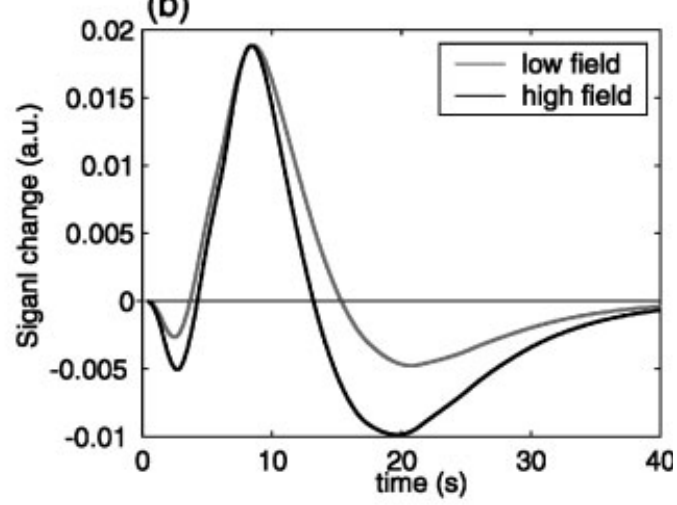

Figure 7. (a) Representation of the $R_{2}{ }^{*}$ space as a function of blood volume $V$ and susceptibility $\chi$. Grey and black lines show iso- $R_{2}{ }^{*}$ contours for $3 \mathrm{~T}$ and $9 \mathrm{~T}$ respectively. The thick black line is the trajectory in this space as calculated from the balloon model as described by Buxton et al. (27). The parameters used were: $\delta_{f}=2, \delta_{m}=6, \tau_{h}=1.3, \tau_{M T T}=4, f_{1}=m_{1}=1.5, \tau^{-}=20, \tau^{+}=10$. (b) shows the shape of the BOLD signal change in arbitrary units in order to depict the difference between the two different field strength regimes. 


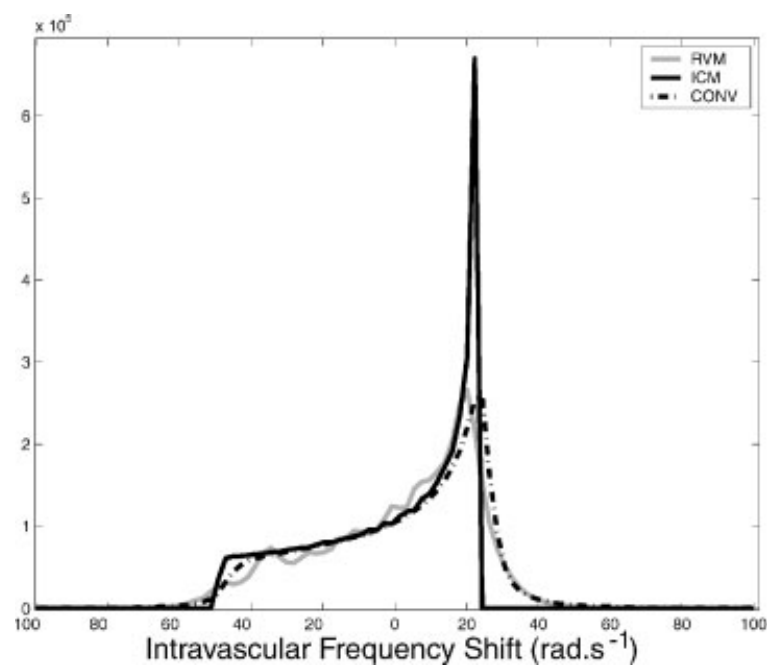

Figure 8. Intravascular frequency shift at $3 \mathrm{~T}$ for a susceptibility difference of $0.18 \mathrm{ppm}$ between intravascular and extravascular compartments. The frequency distribution of the ICM is shown in black, and the distribution obtained for the RVM is shown in grey. The dashed black line represents the frequency distribution produced by convolving the extravascular field distribution with the intravascular distribution expected for the ICM model.

clear that the initial dip should be more visible at higher fields, not only because of the increased contrast expected from the greater field strength, but also because of the different dependencies of $\Delta R_{2}{ }^{*}$ on the changes in blood oxygenation and volume.

\section{Intravascular contrast}

The main difference between ICM and RVM seems to be in the intravascular contribution to BOLD contrast. Figure 8 shows intravascular frequency distributions generated using the ICM (by combining data from 10 different directions of the field, $\vec{B}_{0}$, with respect to the vessel orientation using an isotropic weighting, $\sin \theta$ ) and the RVM (combining data from five different orientations of the model with respect to $B_{0}$ ). As alluded to above, differences between the two models result from field perturbations at junctions between vessels in the RVM. The difference in the distribution can also be partially explained by the fact that vessels in the RVM experience magnetic fields generated by neighbouring vessels as well as the varying field shift due to the range of vessel orientations [eqn (1)]. To assess the magnitude of this effect, we can imagine inserting a cylinder randomly into the extravascular space of the RVM many times, and then assume that the distribution of values inside the cylinder averaged over the different orientations and locations provides a good representation of the distribution of the intravascular frequency shifts. As this cylinder can be sited at any position and with any orientation, the frequency distribution obtained after averaging over the ensemble of locations and orientations can be understood as a convolution of the extravascular frequency distribution from the RVM with the intravascular frequency distribution due to an isotropic distribution of infinite cylinders. The dashed line in Fig. 8 is the result of such a convolution, which shows good qualitative agreement with the grey line, which represents the intravascular field distribution calculated using the RVM.

The simulated intravascular signal produced using the ICM was naturally in very good agreement with eqn (8), once the effect of the $T_{2}$ relaxation of blood was removed. Various attempts were made to fit functions to the variation in the intravascular signal of the RVM with echo time. The approach that proved to be most robust was based on using the intravascular decay curve obtained from the larger length scale (average vessel diameter of $100 \mu \mathrm{m})$ for each $\chi$ and $V$ value, which we write as $I_{V, \chi}(t)$. These curves were subsequently used as models for the data obtained at smaller average vessel diameters, because decay curves obtained at larger length scales are expected to be independent of diffusion. Using such a model, instead of simply using eqn (8), has the advantage of eliminating geometry dependencies of the vascular model that might be introduced by the lack of complete angular averaging, allowing just the effect of blood diffusion within the vascular network to be evaluated. The effect of diffusion was modelled as an exponential multiplier, so that the fitting function became $I_{V, \chi}(t) e^{-R_{2 d i j t} t}$. Figure 9 shows the parameters that characterise the effect of diffusion inside the vessels. The values of $R_{2 \text { diff }}$ are one order of magnitude greater than the $R_{2}{ }^{*}$ values observed in grey matter. Note also that the $R_{2}$ values shown are negative, meaning that diffusion tends to reduce the signal decay because of dephasing of intravascular signal. It can also be expected that flow in the vessels will have a similar, but potentially larger, effect acting to reduce further the attenuation of intravascular signal.

\section{Combining the different contributions to contrast}

As both intravascular and extravascular contributions to BOLD contrast have now been discussed, the next step is to analyse their combined effect. Figure 10 shows the different contributions to the BOLD contrast for the specific case of a blood volume fraction of $1.29 \%$, which does not vary with activation, and a typical change in susceptibility on activation of $0.18 \mathrm{ppm}$, corresponding to a change of $20 \%$ in the blood oxygenation. Figure 10a shows that, at different echo times, different relative intravascular/extravascular contributions to the BOLD signal arise. At echo times varying from 0 to $40 \mathrm{~ms}$, the intravascular contribution to contrast is over-represented (over 10 times greater than its volume fraction in the voxel). The relative intravascular contribution increases for smaller vessels, where diffusion 

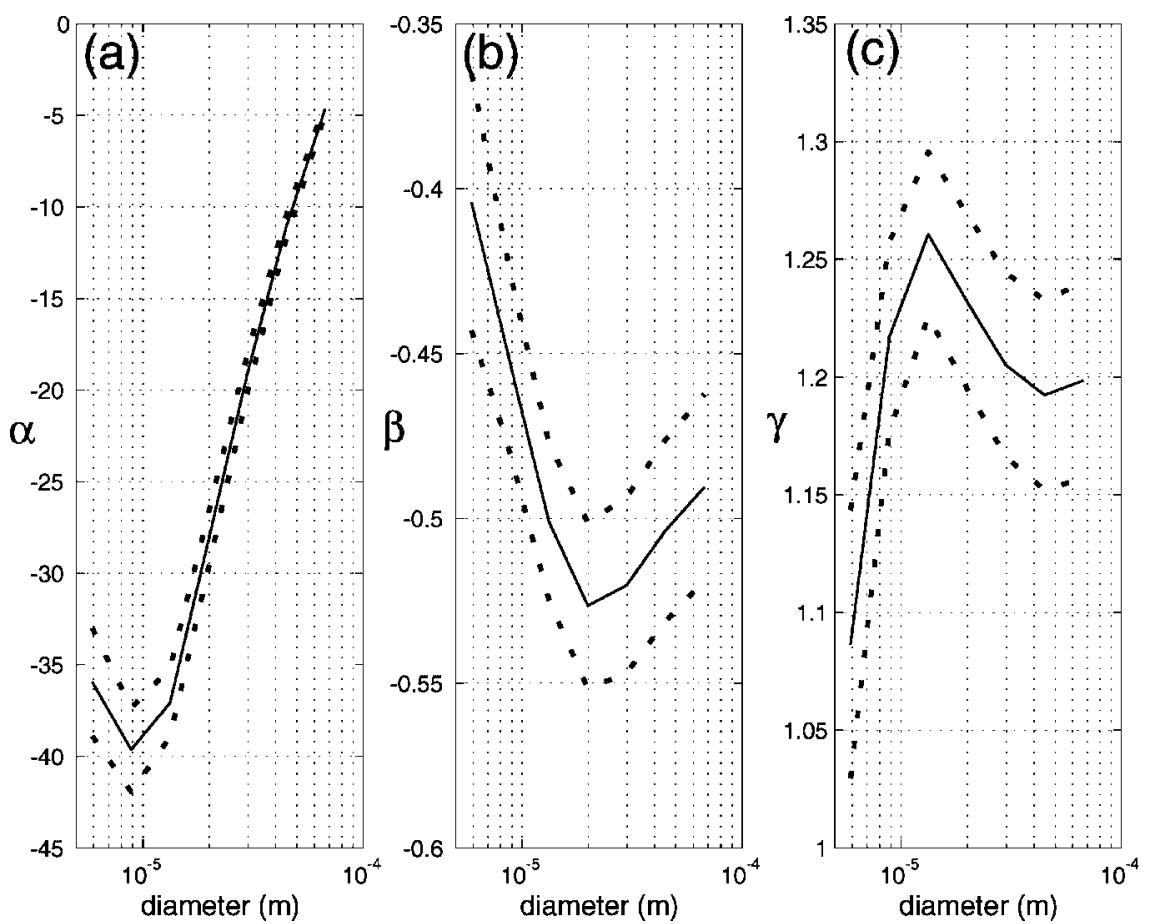

Figure 9. Variation in the parameters (a) $\alpha$, (b) $\beta$ and (c) $\gamma$ (continuous lines) with the average vessel diameter obtained for the ICM (black) and the RVM (grey). In each case, $R_{2}{ }^{*}$ due to diffusion in the gradients present in the intravascular compartment obtained for various values of $V$ and $\chi$ were fitted to a function of the type $\alpha V^{\beta} \chi^{\gamma}$. Dotted lines indicate standard deviations.
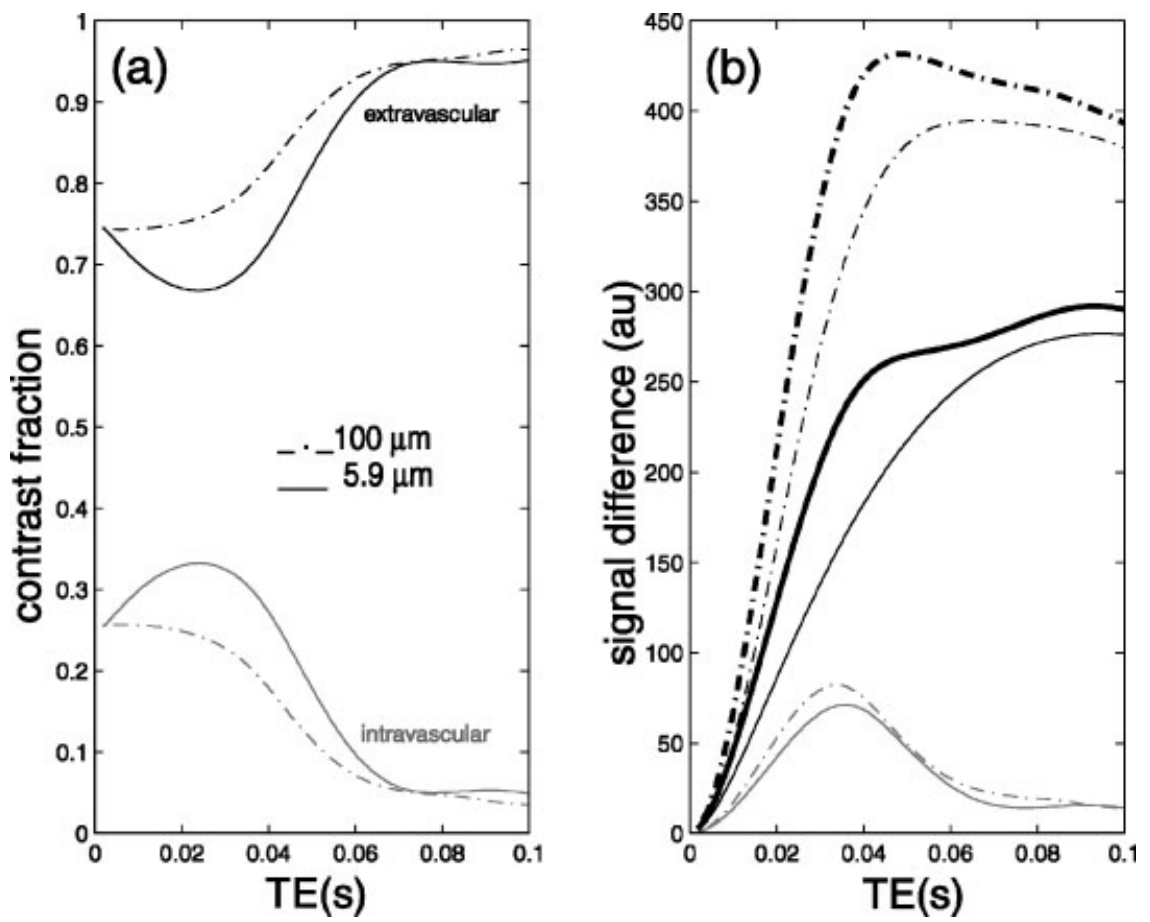

Figure 10. Dependence of the GE BOLD contrast in intravascular and extravascular compartments. (a) The relative contribution to the total contrast in a voxel in which cerebral blood volume $=1.29 \%$ and there is a change of $\chi$ from $0.36 \mathrm{ppm}$ to $0.18 \mathrm{ppm}$ is shown as a function of echo time for extravascular (black) and intravascular (grey) contributions. (b) The time course of the total (thick black), extravascular (black) and intravascular (grey) contrast is shown for the large length scale regime (where the average vessel diameter is $100 \mu \mathrm{m}$; dashed lines) and short length scale regime (where the average vessel diameter is $5.9 \mu \mathrm{m}$; continuous line). 
plays a greater role. The data shown correspond to a field strength of $3 \mathrm{~T}$. The range of echo times for which the relative contribution of the intravascular compartment is significant will decrease with increasing magnetic field strength for two main reasons: $B_{0}$ appears in the argument of the Bessel function series of eqn (8); $R_{2}{ }^{*}$ of deoxygenated blood also increases with field.

The different contributions to the overall relaxation of the signal following a GE sequence, which have been parameterised throughout this paper, can be condensed into the following expression:

$$
\begin{aligned}
\operatorname{Signal}(t, V, \Delta \chi)= & M_{0, G M} \frac{100-V}{100} e^{-R_{2, G M} t} e^{-2.73 V^{1.1} \chi^{1.3} t} \\
& +M_{0, B} \frac{V}{100} I(t) \times e^{-3.5 V^{1} \chi^{1.2 t}} \times e^{40 V^{-0.4} \chi^{1.2} t}
\end{aligned}
$$

where the term in the first line refers to the extravascular contribution to the signal, and the term in the second line refers to the intravascular contribution. The latter has various contributions, as discussed in the Methods section: (a) $I(t)$ refers to the isotropically oriented vessels; $(b)$ refers to static dephasing, which, considering the argument presented in Fig. 8, should be the same as for grey matter at the static regime (Fig. $4 a-c) ;(c)$ is an averaged effect of that shown in Fig. 9 based on the same reasoning as used to collapse the various length scale dependencies for the extravascular $R_{2}{ }^{*}$ contrast.

Equation (11) can be rewritten as a function of the more meaningful parameters, oxygenation fraction, $Y$, static magnetic field, $B_{0}$, and blood volume percentage, $V$ :

$$
\begin{array}{r}
\text { Signal }\left(t, V, Y, B_{0}\right)=M_{0, G M} \frac{100-V}{100} e^{-R_{2, G M} t} e^{-0.57 V^{1.1}(1-Y)^{1.3} B_{0}^{1.3} t} \\
+M_{0, B} \frac{V}{100} I\left(t, Y, B_{0}\right) \times e^{-\left(0.83 V^{1}-9.5 V^{-0.4}\right)(1-Y)^{1.2} B_{0}^{1.2} t}
\end{array}
$$

To account totally for the intravascular contrast it would be also necessary to simulate the effect of diffusion of water around the red blood cells, which would be expected to introduce a factor $e^{-R_{2, \text { bloodPOOL }}^{*}(\mathrm{X}) t}$ multiplying the intravascular term of eqns (11) and (12). Such a procedure would be feasible, using the same approach as used for the realistic vasculature model. However, there are two problems that prevent the straightforward implementation of such an approach: the time steps required to simulate the effect of diffusion would be extremely small, making the simulation even more time consuming; the red blood cell volume fraction in blood is $\sim 40 \%$ (much larger than the partial volume of blood in grey matter, $\sim 4 \%$ ) and the field shifts for such a high packing fraction are extremely dependent on the arrangement of the blood cells (in preliminary simulations, arrangements such as cubic, cubic centred, and hexagonal configurations showed very different distributions of frequency shifts). Another reason for not simulating the intravascular contrast dependence on red blood cell concentration and oxygenation is that such dependence can be measured in vitro with much greater reliability (18).

\section{DISCUSSION}

Most of the results presented in this paper imply that the ICM provides a good description of the extravascular contrast, as is shown by the close agreement between the parameters obtained using the RVM and ICM, which is an important conclusion in itself. The parameterisation of the extravascular relaxivity shows that the assumption of a linear dependence of relaxation rate on blood volume in functional studies using contrast agents is a good approximation, but is not necessarily valid for endogenous contrast agents, mostly because of the non-exponential decay that characterises the initial FID. Although the dependence found for $\Delta R_{2}{ }^{*}$ on the susceptibility difference, in the static regime, and on the blood volume fraction, in both the static and diffusion-narrowing regimes, is not linear as expected (4-6,9), this dependence can be understood by considering the manner in which the curve fitting was performed. The $\Delta R_{2}{ }^{*}$ values used for the parameterisation in this paper were calculated considering a fixed range of echo times ( 0 to $80 \mathrm{~ms}$ ), as used in most experiments directed towards making quantitative estimates of $\Delta R_{2}{ }^{*}((28,29)$, for example), where one or multiple echo times spread at fixed intervals through the signal decay are used. Therefore, in these simulations, by fitting an exponential to a non-exponential decay, in a finite time range, although the non-exponential decay width scales linearly, for example in the static regime with various values of susceptibility, the value of the exponential relaxation rate will not scale linearly. For this rate to scale linearly, the fitting region of the fit would have to be varied according to the width change. With these limitations in mind, it is interesting to note that the dependence of $R_{2}{ }^{*}$ on the susceptibility, $\chi$, has the expected trend: values of $\gamma$ vary from close to 2 in the diffusion-narrowing regime to values closer to 1 in the static regime.

Despite the agreement, the use of a realistic model, as opposed to infinite cylinders, is relevant, as capillary positions are indeed correlated (30), which can be understood as an attempt to optimise the homogeneity of the oxygen supply (11). The use of the Fourier method for the calculation of the frequency shift (14) of the model is also essential when dealing with the realistic model, as otherwise the discussions would have to be limited to mutually avoiding cylinders as considered in other studies (11). Use of RVMs rather than the ICM for calculating the relaxation effects of the vasculature may be particularly important in the future for studies of tumours, where the blood volume fraction may be much higher than that considered here. 
Using a realistic model of the vasculature to simulate the BOLD effect has brought some new insight to the importance of different contributions to the overall BOLD contrast, as can be found parameterised in eqns (11) and (12). One interesting result is the greater role of intravascular contrast in small vessels, which is not only due to the increased diffusion effects in the extravascular tissue surrounding capillaries, as extensively described in the literature $(3,10)$. The effect of flow should also be evaluated in future work. Preliminary studies indicate that flow causes a line-narrowing of the intravascular signal, which can be understood as a reduction in the phase accumulation experienced by each individual spin because of flowing through the network of vessels in which there is a significant distribution of field shifts.

\section{Acknowledgements}

This work was supported by Fundação para a Ciência e Tecnologia via the scholarships SFRH/BD/909/2000 and SFRH/BPD/20522/2004 to J.P.M. and by the MRC grant G9900259.

\section{REFERENCES}

1. Ogawa S, Lee TM, Kay AR, Tank DW. Brain magneticresonance-imaging with contrast dependent on blood oxygenation. Proc. Natl. Acad. Sci. 87: 9868-9872. 1990.

2. Ogawa S, Lee TM, Barrere B. The sensitivity of magnetic resonance image signals of a rat brain to changes in the cerebral venous blood oxygenation. Magn. Reson. Med. 29: 205-210. 1993.

3. Ogawa S, Menon RS, Tank DW, Kim SG, Merkle H, Ellermann JM, Ugurbil K. Functional brain mapping by blood oxygenation level-dependent contrast magnetic resonance imaging. A comparison of signal characteristics with a biophysical model. Biophys. J. 64: 803-812. 1993.

4. Yablonskiy DA, Haacke EM. Theory of the NMR signal behavior in magnetically inhomogeneous tissues: the static dephasing regime. Magn. Reson. Med. 32: 749-763. 1994.

5. Boxerman JL, Hamberg LM, Rosen BR, Weisskof RM. MR contrast due to intravascular magnetic susceptibility perturbations. Magn. Reson. Med. 34: 555-566. 1995.

6. Kiselev VG, Posse S. Analytical model of susceptibility-induced MR signal dephasing: effect of diffusion in a microvascular network. Magn. Reson. Med. 41: 499-509. 1999.

7. Motti EDF, Imhof HG, Yasargil MG. The terminal vascular bed in the superficial cortex of the rat. An SEM study of corrosion casts. J. Neurosurg 65: 834-846. 1986.

8. Bandettini PA, Wong. EC. The effects of biophysical and physiologic parameters on brain activation-induced $r_{2}{ }^{*}$ and $r_{2}$ changes: simulations using a deterministic diffusion model. Int J Imaging Syst Technol 6: 133-152. 1995.

9. Kiselev VG, Posse S. Analytical theory of susceptibility induced NMR signal dephasing in a cerebrovascular network. Phys. Rev. Lett. 81: 5696-5699. 1998.
10. Fujita N. Extravascular contributions of blood oxygenation leveldependent signal changes: a numerical analysis based on a vascular network model. Magn. Reson. Med. 46: 723-734. 2001.

11. Kiselev VG. Effect of magnetic field gradients induced by microvasculature on NMR measurements of molecular self-diffusion in biological tissues. J. Magn. Reson. 170: 228-235. 2004.

12. Buxton RB, Wong EC, Frank LR. Dynamics of blood flow and oxygenation changes during brain activation: the balloon model. Magn. Reson. Med. 39: 855-864. 1998.

13. Weisskoff R, Kiihne S. MRI susceptometry: image-based measurements of absolute susceptibility of MR contrast agents and human blood. Magn. Reson. Med. 24: 375-383. 1992.

14. Marques JP, Bowtell RW. Application of a Fourier-based method for rapid calculation of field inhomogeneity due to spatial variation of magnetic susceptibility. Concepts in Magnetic Resonance 25B: 65-78. 2005.

15. Salomir R, de Senneville BD, Moonen CTW. A fast calculation method for magnetic field inhomogeneity due to an arbitrary distribution of susceptibility. Concepts in Magnetic Resonance 19B: 26-34. 2003.

16. Crank J. The Mathematics of Diffusion, 2nd edition. Oxford Science Publications: Oxford, 1975.

17. Wansapura JP, Holland SK, Dunn RS, Ball. WS. NMR relaxation times in the human brain at 3.0 Tesla. J. Magn. Reson. Imaging 9: 531-538. 1999.

18. Spees WM, Yablonskiy DA, Oswood MC, Ackerman JH. Water proton MR properties of human blood at 1.5 Tesla: magnetic susceptibility $T 1, T 2, T_{2}{ }^{*}$ and non-Lorentzian signal behavior. Magn. Reson. Med. 45: 533-542. 2001.

19. Kennan RP, Zhong J, Gore. JC. Intravascular susceptibility contrast mechanisms in tissues. Magn. Reson. Med. 31: 9-21. 1994.

20. He X, Yablonskiy DA. Quantitative BOLD: mapping of human cerebral deoxygenated blood volume and oxygen extraction fraction: default state. Magn. Reson. Med. 57: 115-126. 2007.

21. Kjølby BF, Østergaard L, Kiselev VG. Theoretical model of intravascular paramagnetic tracers effect on tissue relaxation. Magn. Reson. Med. 56: 187-197. 2006.

22. Ziener CH, Kampf T, Melkus G, Jakoband PM, Bauer WR. scaling laws for transverse relaxation times. J. Magn. Reson. 184: 169-175. 2007.

23. Fisel CR, Ackerman JL, Buxton RB, Garrido L, Belliveau JW, Rosen BR, Bardy TJ. MR contrast due to microscopically heterogeneous magnetic susceptibility: numerical simulations and applications to cerebral physiology. Magn. Reson. Med. 17: 336-347. 1991.

24. Turner R. How much cortex can a vein drain? Downstream dilution of activation-related cerebral blood oxygenation. Neuroimage, 16: 1062-1067. 2002.

25. Yacoub E, Shmuel A, Pfeuffer J, Van de Moortele P-F, Adriany G, Ugurbil K, Hu. X. Investigation of the initial dip in fMRI at 7 Tesla. NMR Biomed, 44: 408-412. 2001.

26. Yacoub E, Hu. X. Detection of the early negative response in fMRI at 1.5T Tesla. Magn. Reson. Med. 41: 1088-1092. 1999.

27. Buxton RB, Uludăg K, Dubowitz DJ, Liu. TT. Modeling the hemodynamic response to brain activation. Neuroimage, 23: S220-S233. 2004.

28. Cohen R, Rostrup E, Sidaros K, Lund TE, Paulson Olaf B., Ugurbil Kamil, Kim Seong-Gi. Hypercapnic normalization of BOLD fMRI: comparison across field strengths and pulse sequences. NeuroImage 23: 613-624. 2004.

29. Posse S, Kemna LJ, Elghahwagi B, Wiese S, Kiselev VG. Effect of graded hypo- and hypercapnia on fMRI contrast in visual cortex: Quantication of $T_{2}{ }^{*}$ changes by multiecho EPI. Mag. Reson. Med. 46: 264-271. 2001.

30. Pawlik G, Rackl A, Bing. RJ. Quantitative capillary topography and blood flow in the cerebral cortex of cats: an in vivo microscopic study. Brain Res. 208: 3558. 1981. 